\title{
Simultaneous determination of amlodipine and telmisartan from pharmaceutical products by way of capillary electrophoresis
}

\author{
Modroiu Adriana, Hancu Gabriel ${ }^{\star}$, Vlad Robert Alexandru, \\ Stăcescu Ștefana, Soare Ruxandra, Kelemen Hajnal
}

Department of Pharmaceutical Chemistry, Faculty of Pharmacy, University of Medicine and Pharmacy Tîrgu Mureş, Gh. Marinescu 38, 540139 Tîrgu Mureş, Romania

\section{ARTICLE INFO \\ Received 16 November 2016 \\ Accepted 02 March 2016}

\section{Keywords:}

fixed-dose combinations,

Amlodipine,

Telmisartan,

capillary electrophoresis,

simultaneous determination.

\begin{abstract}
A rapid, simple and sensitive capillary zone electrophoresis method was developed for the simultaneous determination of amlodipine besylate and telmisartan, in fixed-dose combinations, through the utilization of a UV photodiode array detector. Electrophoretic parameters such as buffer concentration and $\mathrm{pH}$, system temperature, applied voltage and injection parameters, wereoptimized in order to improvetheefficiencyof theseparation. The best results were obtained when employing fused silica capillary ( $48 \mathrm{~cm}$ length X $50 \mu \mathrm{m}$ ID) and $50 \mathrm{mM}$ phosphate buffer electrolyte at $\mathrm{pH} 4.50,+25 \mathrm{kV}$ applied voltage, as well as $25^{\circ} \mathrm{C}$ system temperature. The separation was achieved in approximately 3 minutes, with a resolution of 4.90 , while the order of migration was amlodipine followed by telmisartan. The analytical performance of the method was verified with regard to linearity, precision and robustness. In addition, the limit of detection and the quantification were calculated.
\end{abstract}

Hypertension (also known as arterial hypertension or high blood pressure) is currently the biggest single contributor to global mortality, and extensive randomized trial data are consistent in showing that blood pressure reduction substantially reduces cardiovascular morbidity and mortality [11].

Since it is very difficult to control blood pressure using a single antihypertensive agent, and an adequate blood pressure control is often made possible among the majority of patients only if combinations of $\geq 2$ antihypertensive medications are used for treatment; attempts have been made in recent years to create a more ideal treatment for hypertension by combining complementary drugs in fixed dose combinations [10]. The rationale behind fixed dose combination therapy is that the use of two complementary agents in lower doses that are with fewer dose-dependent side effects, makes it possible to obtain potential benefits attributable to possible synergistic pharmacological and physiological actions, as well as to engender better patient compliance and adherence towards treatment [3].

The combinations of a renin-angiotensin-aldosterone system-targeting agents, such as angiotensin-converting-

\footnotetext{
* Corresponding author

e-mail: gabriel.hancu@umftgm.ro
}

enzyme (ACE) inhibitors or angiotensin II receptor blockers (ARB), and diuretic or calcium channel blockers (CCB), appears to provide synergy with regard to blood pressure lowering [15].

One such combination is the one between ARB telmisartan (TEL) and CCB amlodipine (AML). This has demonstrated significantly greater blood pressure reductions compared with the utilization of each monotherapy component, in the overall population, and, in particular, in patients with moderate to severe hypertension, as well as high-risk patients [12].

Taking into consideration the great prevalence and importance of fixed dose combination in modern therapy, the elaboration of new methods of analysis for the simultaneous determination of combined substances represents a necessity and also a challenge for the analysts.

The large majority of methods, described in literature, for the simultaneous determination of antihypertensive drugs from fixed combinations, utilize high performance liquid chromatography (HPLC); but capillary electrophoresis (CE), due to its advantages related to short-analysis time, rapid method development and low consumption of analytes and reagents, has emerged in recent years as an alternative and also a complementary method to the more frequently employed chromatographic procedures. 
Literature review reveals various methods for the determination of AML and TEL, individually or in combination, from pharmaceutical products, by RP-HPLC $[9,13]$, HPTLC [2] and UV spectrophotometry [7,8,14].

To our knowledge, no CE method for the simultaneous determination of AML and TEL has been published so far. However, CE has been used for the simultaneous measurement of AML and atorvastatine [5] and of TEL and hydrochlorthiazide [6] from their combination drug formulations. Also a CE method was developed for the simultaneous determination of AML and another ARB, valsartan, in pharmaceuticals and human plasma [1], and, more recently, CE was applied for the simultaneous determination in tablets of the triple combination: AML, valsartan and hydrochlorthiazide [4].

Our aim was to bring forth the development of a simple, rapid and efficient $\mathrm{CE}$ method for the simultaneous determination of AML and TEL, as well as the optimization of analytical conditions, and to verify the applicability of the newly developed method for the determination of the two analytes from pharmaceutical products.

\section{EXPERIMENTAL}

\section{Chemicals}

Pharmaceutical grade samples of amlodipine besylate (Orex Pvt. Ltd, India) and telmisartan (RA ChemPharma Limited, Hyderabad, India) were employed in the study. The phosphoric acid, disodium hydrogenophosphate, sodium didydrogenophosphate were purchased from Merck (Germany), while sodium hydroxide and methanol were obtained from LachNer (Czech Republic). All reagents were of analytical purity. Purified water was provided by a Milli-Q Plus water purification system (Millipore, USA). The pharmaceutical dosage forms investigated in this study were Twynsta (Boehringer Ingelheim International $\mathrm{GmbH}$, Germany), containing a TEL/AML besylate ratio of $40 / 5$ and $80 / 10 \mathrm{mg}$, respectively.

\section{Equipment}

All measurements were performed on an Agilent $1600 \mathrm{CE}$ system equipped with a photodiode array (DAD) detector, with Chemstation software used for data handling. Separations were performed on an uncoated fused-silica capillary with a total length of $48 \mathrm{~cm}$ (40 cm effective length), having an internal diameter of $50 \mu \mathrm{m}$ (Agilent, Germany). Buffer $\mathrm{pH}$ was determined using a Terminal 740 pH-meter (Inolab, Germany).

\section{Electrophoretic procedure}

Conditioning of new capillaries was conducted by flushing with $0.1 \mathrm{M} \mathrm{NaOH}$ for 60 minutes, and then water for 30 minutes. Prior to all runs, the capillary was preconditioned by flushing with $0.1 \mathrm{M} \mathrm{NaOH}$, water, and background electrolyte (BGE) - each for 2 minutes.

Different phosphate and borate buffers were tested in a $\mathrm{pH}$ range between 2-11. BGE solutions were prepared by dissolving the appropriate amount of buffer constituents in ultrapure water and adjusting the $\mathrm{pH}$ (if necessary) with $1 \mathrm{M}$ $\mathrm{H}_{3} \mathrm{PO}_{4}$ or $1 \mathrm{M} \mathrm{NaOH}$ solutions. Stock solutions containing
$1 \mathrm{mg} \mathrm{mL}^{-1}$ of each analyte were prepared in methanol and diluted prior to use with the same solvent, to the appropriate concentration. Both BGE and sample solutions were filtered through a $0.45 \mu \mathrm{m}$ pore size membrane filter and sonicated in an ultrasonic bath for 5 minutes prior to use.

In the preliminary analysis, we applied some "standard" electrophoretic conditions for a $\mathrm{CE}$ analysis: temperature $20^{\circ} \mathrm{C}$, applied voltage $+20 \mathrm{kV}$, injection pressure $\mathrm{x}$ time 50 mbar x 3 second, sample concentration $25 \mu \mathrm{g} \mathrm{mL}-1$. The samples were introduced in the system at the anodic end of the capillary by hydrodynamic injection. Detection was performed at 210, 230 and $250 \mathrm{~nm}$, and full spectra of the analytes were also stored to facilitate peak identification.

When preparing samples drawn from commercial formulations, their average weight was first calculated by weighing 20 tablets. These were then crushed, mixed and powdered in a mortar into a homogenous powder. Subsequently, an amount of powder equivalent to the weight of one tablet was dissolved in $100 \mathrm{~mL}$ methanol by sonication for 5 minutes, with intermittent shaking. Following this, the solution was filtered through a $0.45 \mu \mathrm{m}$ syringe, centrifuged at $3500 \mathrm{rpm}$ for 10 minutes and diluted with methanol to the appropriate concentration. The same procedure was applied as in the separation from standard solutions.

\section{RESULTS AND DISCUSSON}

\section{Optimization of the analytical conditions}

The chemical structure of the two analytes: AML(RS)3-ethyl 5-methyl 2-[(2-aminoethoxy)methyl]-4-(2chlorophenyl)-6-methyl-1,4-dihydropyridine-3,5-dicarboxylate) and TEL (2-(4-\{[4-Methyl-6-(1-methyl-1H-1,3-benzodiazol-2-yl)-2-propyl-1H-1,3-benzodiazol-1-yl]methyl\} phenyl)benzoic acid) are presented in Figure 1.

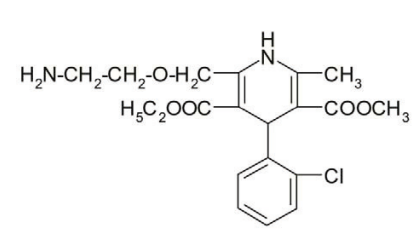

Amlodipine (AML)

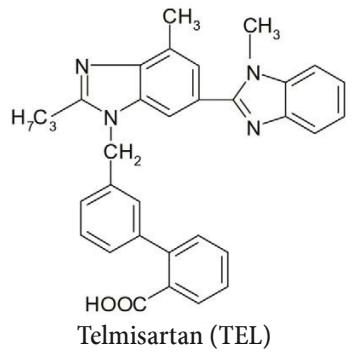

Telmisartan (TEL)
Figure 1. Chemical structure of the studied analytes

The $\mathrm{pH}$ value is always a critical selectivity parameter for the determination of acidic and basic compounds. This because it determines the degree of the analytes ionization, their electrophoretic mobility and the magnitude of the electro-osmotic flow (EOF). In our case, AML is a weak basic compound with a pKa value of 8.7 , being characterized by the presence of a ionizable amino $\left(-\mathrm{NH}_{2}\right)$ group, while TEL, with pKa values of 3.5 , has a ionizable carboxylic (-COOH) group with pKa values of 3.5 and 6.0 [1,13]. Under acidic conditions, both AML and TEL are positively charged, but as the $\mathrm{pH}$ becomes more alkaline, their effective mobility decreases. Herein, AML starting to migrate vey close to the EOF. Both analytes can be detected simultaneously over a large $\mathrm{pH}$ range $(3.5-8.0)$, but the best correlation between 
migration times, resolution and peak shapes was obtained when using a phosphate buffer at $\mathrm{pH} 4.50$.

Buffer concentrations between 25 and $100 \mathrm{mM}$ were studied at a constant $\mathrm{pH}$ of 4.50 . In our work, a $50 \mathrm{mM}$ phosphate buffer was chosen because it generated good peak shapes, short migration times and stable baseline current, while greater buffer concentrations brought about longer migration times and high currents which generated electrophorettic system instability.

In order to determine the optimal voltage to be applied, the influence of voltage $(10-30 \mathrm{kV})$ on migration times and resolution was investigated under the optimized BGE conditions; a voltage of $+25 \mathrm{kV}$ was selected as the optimum. Application of high voltages is limited by the Joule effect, and, consequently, by the electrophoretic system capacity to dissipate generated heat.

The influence of capillary temperature $\left(15-25^{\circ} \mathrm{C}\right)$ was evaluated under the chosen BGE conditions; when temperature increased, migration times decreased; a temperature of $25^{\circ} \mathrm{C}$ was chosen as working temperature for the analysis.

In order to determine the optimal injection parameters, the influences of injection time (1-5 s) and injection pressure (25-50 mbar) were studied in order to attain lower detection limits, while not affecting the peak shape, migration time and resolution. An injection pressure of $50 \mathrm{mbar}$ and an injection time of 2 seconds were selected.

The best results for the simultaneous determination of AML and TEL were obtained when using $50 \mathrm{mM}$ phosphate buffer at $\mathrm{pH} 4.50,+25 \mathrm{kV}$ applied voltage, $25^{\circ} \mathrm{C}$ system temperature, injection pressure $50 \mathrm{mbar}$, injection time 2 seconds, UV detection at $210 \mathrm{~nm}$. The two analytes migrated in less than 3 minutes, the order of migration was AML, followed by TEL; the resolution of the separation was 4.90 - with a selectivity factor of 1.09 (Fig. 2).

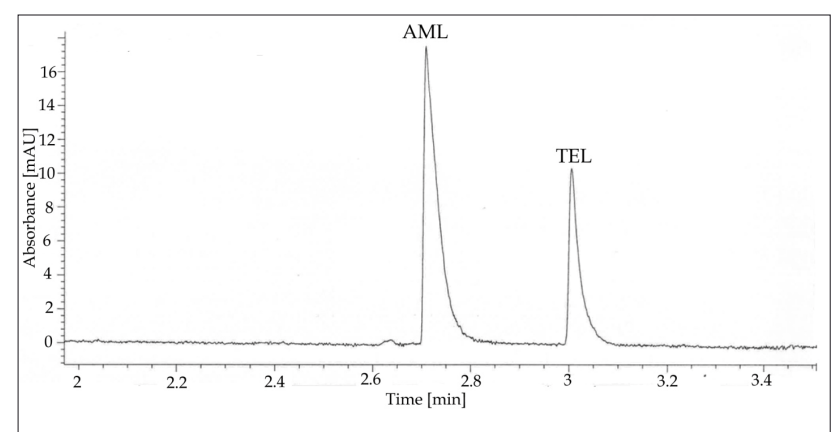

Figure 2. Typical electropherogram of the AML-TEL simultaneous separation under optimized conditions (buffer: $50 \mathrm{mM}$ phosphate, $\mathrm{pH}-4.50$, voltage: $+25 \mathrm{kV}$, capillary temperature: $25^{\circ} \mathrm{C}$, injection pressure/time: $50 \mathrm{mbar} / 2 \mathrm{~s}$, UV detection at $210 \mathrm{~nm}$, analyte concentrations: $25 \mu \mathrm{g} \mathrm{mL}^{-1}$ )

\section{Analytical performance}

Linearity solutions were prepared from stock solution at six concentration levels and three replicates per concentration. The linear regression analysis of AML and TEL were constructed by plotting the peak area of the analytes (y) versus analyte concentrations $(\mathrm{x})$. The calibration curves were linear in the studied range $\left(5-100 \mu \mathrm{g} \mathrm{mL}^{-1}\right.$ for TEL, 1-25 $\mu \mathrm{g} \mathrm{mL}^{-1}$ for AML), with correlation coefficients above 0.99 . The regression equation and correlation coefficients are presented in Table 1.

Table 1. Analytical parameters for the simultaneous determination of AML and TEL $(n=6)$

\begin{tabular}{|l|c|c|c|c|c|}
\hline Analyte & $\begin{array}{c}\text { Concentration } \\
\text { range } \\
\left(\mu \mathrm{mL}^{-1}\right)\end{array}$ & $\begin{array}{c}\text { Regression } \\
\text { equation }\end{array}$ & $\begin{array}{c}\text { Correlation } \\
\text { coefficient }\end{array}$ & $\begin{array}{c}\mathrm{LOD} \\
\left(\mu \mathrm{gL}^{-1}\right)\end{array}$ & $\begin{array}{c}\mathrm{LOQ} \\
\left(\mu \mathrm{g} \mathrm{mL}^{-1}\right)\end{array}$ \\
\hline AML & $1-25$ & $\begin{array}{c}\mathrm{y}=0.318 \mathrm{x} \\
+10.39\end{array}$ & 0.9916 & 0.66 & 2.18 \\
\hline TEL & $5-100$ & $\begin{array}{c}\mathrm{y}=0.166 \mathrm{x} \\
+4.714\end{array}$ & 0.9907 & 1.14 & 3.42 \\
\hline
\end{tabular}

An approach based on the standard deviation of the response and the slope of the calibration plots was used to determine detection (LOD) and quantification limits (LOQ). LOD and LOQ values were estimated as [(standard deviation of repeatability)/(slope of the regression equation)] by multiplying with 3.3 and 10, respectively. The values obtained are given in Table 1.

Intra-day precision was assessed by obtaining the relative standard deviation (RSD) of five repeated measurements of samples at the three concentration levels. Inter-day precision was determined by analyzing the same set of samples for three different days. The RSD values indicate good precision of the method (Table 2).

Table 2. Intra and inter-day precision for the simultaneous determination of AML and TEL

\begin{tabular}{|c|c|c|c|c|}
\hline \multirow{3}{*}{$\begin{array}{l}\text { Analyte concentration } \\
\qquad\left(\mu \mathrm{g} \mathrm{mL}^{-1}\right)\end{array}$} & \multicolumn{4}{|c|}{ RSD (\%) } \\
\hline & \multicolumn{2}{|c|}{ Migration time } & \multicolumn{2}{|c|}{ Peak area } \\
\hline & AML & TEL & AML & TEL \\
\hline \multicolumn{5}{|l|}{ Intra-day precision $(n=5)$} \\
\hline 5 & 0.11 & 0.22 & 1.11 & 1.25 \\
\hline 15 & 0.12 & 0.22 & 0.86 & 1.30 \\
\hline 25 & 0.12 & 0.24 & 0.90 & 1.27 \\
\hline \multicolumn{5}{|l|}{ Inter-day precision $(n=15)$} \\
\hline 5 & 0.44 & 0.87 & 1.68 & 2.16 \\
\hline 15 & 0.50 & 0.90 & 1.79 & 2.14 \\
\hline 25 & 0.48 & 0.94 & 1.74 & 2.11 \\
\hline
\end{tabular}

To demonstrate the robustness of the method, minor changes in the experimental conditions were made. Here, the $\mathrm{pH}$ of the buffer was varied in the range $\pm 0.5 \mathrm{pH}$ unit, separation temperature was altered in the range $\pm 2{ }^{\circ} \mathrm{C}$, while applied voltage was modified in the range $\pm 2 \mathrm{kV}$. None of the modifications caused significant changes in the resolution between the drugs. In this work, RSD for migration times and peak areas was under $2 \%$.

The solution stability of AML and TEL was carried out by leaving the test solution in tightly capped volumetric flask at room temperature for 24 hours. The sample solution was then assayed against freshly prepared standard solutions. In our case, the RSD of the assay of AML and TEL were within $5 \%$. This indicates that both standard and test preparation were stable for 24 hours, while standing upon bench top at room temperature.

Accuracy studies were performed by weighing an appropriate amount of Twynsta tablet powder and spiking it with known amount of the standard compounds, the resulting mixtures were analyzed in triplicate. Accuracy values ranged 
from 98.35 to $101.84 \%$ for AML and from $100.25 \%$ to $103.08 \%$ for TEL, the recoveries demonstrating the good accuracy of the proposed method.

The electropherogram obtained from the sampled tablets were similar to those obtained from standard solutions. Moreover, there was no interference from drug formulation excipients. Furthermore, the $\%$ recovery was found to be between the range $98-102 \%$ - indicating high degrees of accuracy being made possible through utilizing the proposed method (Table 3 ).

Table 3. Assay results of AML and TEL simultaneous determination in pharmaceutical formulations

\begin{tabular}{|l|c|c|c|c|c|c|}
\hline \multirow{2}{*}{$\begin{array}{c}\text { Pharmaceutical } \\
\text { preparation }\end{array}$} & \multicolumn{2}{|c|}{$\begin{array}{c}\text { Declared amount } \\
(\mathrm{mg})\end{array}$} & \multicolumn{2}{|c|}{$\begin{array}{c}\text { Found amount } \\
(\mathrm{mg})\end{array}$} & \multicolumn{2}{|c|}{$\begin{array}{c}\text { RSD } \\
(\%)\end{array}$} \\
\cline { 2 - 7 } & AML & TEL & AML & TEL & AML & TEL \\
\hline Twynsta 40/5 & 5 & 40 & 4.90 & 40.80 & 0.56 & 0.68 \\
\hline Twynsta 80/10 & 10 & 80 & 9.80 & 80.95 & 0.67 & 0.85 \\
\hline
\end{tabular}

\section{CONCLUSIONS}

A CZE method for the simultaneous determination of AML and TEL was successfully developed. Under the optimized conditions, baseline separation of the two analytes was obtained in approximately 3 minutes. Good analytical performance with regards to linearity, precision, reproductibility and robustness was also achieved. What is more, the proposed method was successfully applied for the determination of the analytes in their co-formulated tablets. When compared to previously developed HPLC methods $[9,13]$, the developed method exhibits (as expected) less sensitivity, but, nevertheless, provides shorter migration times and higher separation efficiency. The developed method can, therefore, be used as quality control protocols in pharmaceutical industries.

\section{REFERENCES}

1. Alnajjar A.O.: Validation of a capillary electrophoresis method for the simultaneous determination of amlodipine besylate and valsartan in pharmaceuticals and human plasma. J. AOAC Int., 94, 498, 2011.

2. Chabukswar A.R. et al.: Simultaneous HPTLC estimation of telmisartan and amlodipine besylate in tablet dosage form. Arch. Appl. Sci. Res., 2, 94, 2010.

3. Chrysant S.G.: Using fixed-dose combination therapies to achieve blood pressure goals. Clin. Drug Investig., 28, 713, 2008.

4. Ebeid W. et al.: Simultaneous determination of valsartan, amlodipine besylate and hydrochlorothiazide using capillary zone electrophoresis (CZE). Pharmazie, 70, 368, 2015.

5. Hefnawy M.M., Sultan M., Al-Johar H.: Development of capillary electrophoresis technique for simultaneous measurement of amlodipine and atorvastatin from their combination drug formulations. J. Liq. Chrom. Rel. Technol., 32, 2923, 2009.

6. Hillaert S., van de Bossche W.: Simultaneous determination of hydrochlorothiazide and several angiotensin-II-receptor antagonists by capillary electrophoresis. J. Pharm. Biomed. Anal., 31, 329, 2003.

7. Hirpara K.P. et al.: UV spectrophotometric determination for simultaneous estimation of amlodipine besylate and telmisartan in combination. J. Pharm. Sci. Bio. Res., 2, 133, 2012.

8. Kondawar M.S. et al.: UV spectrophotometric estimation of amlodipine besylate and telmisartan in bulk drug and dosage form by multiwavelenght analysis. Int. J. Pharm. Tech. Res., 3, 1274, 2011.

9. Rajeswari A. et al.: RP-HPLC method development and validation for simultaneous estimation of amlodipine besylate and telmisartan in tablet dosage forms. Int. J. Pharm. Res. Anal., 3, 13, 2013.

10. Ram C.V.: Fixed-dose triple-combination treatments in the management of hypertension. Manag. Care, 22, 45, 2013.

11. Rowan C.G. et al.: Antihypertensive treatment and blood pressure control relative to hypertension treatment guidelines. Pharmacoepidemiol. Drug. Saf., 23, 1294, 2014.

12. Segura J., Ruilope L.M.: Clinical utility of fixed-combination telmisartan-amlodipine in the treatment of hypertension. Integr. Blood Press. Control., 4, 27, 2011.

13. Suresh Kumar G.V., Rajendraprasad Y.: Development and validation of reversed-phase HPLC method for simultaneous estimation of telmisartan and amlodipine in tablet dosage form. Int. J. Pharm. and Pharm. Sci. 2, 128, 2010.

14. Thomas A.B. et al.: Simultaneous spectrophotometric estimation of amlodipine besylate and telmisartan in tablet dosage form. Int. J. Pharm. Tech. Res., 2, 1334, 2010.

15. Wan X., Ma P., Zhang X.: A promising choice in hypertension treatment: Fixed-dose combinations. Asian J. Pharm. Sci., 9, 1, 2014. 
